\title{
GENERATION OF FREQUENCY SHIFTED PICOSECOND PULSES WITH LOW TEMPORAL JITTER
}

\author{
H.J. POLLAND and W. ZINTH \\ Physik Department der Technischen Universität München, Munchen, Fed. Rep. Germany
}

Received 16 February 1984

\begin{abstract}
Transient stimulated Raman scattering is used for the generation of a frequency shifted picosecond light pulse; part of this Raman shifted pulse is subsequently coherently scattered at a material excitation of a second Raman cell. Starting with the second harmonic pulse $\left(t_{\mathrm{p}}=4 \mathrm{ps}\right)$ of a mode-locked $\mathrm{Nd}$ : glass laser system, both the stimulated and the coherently produced pulses have durations of 2.3 ps at different wavelengths. By the appropriate choice of the Raman medium pulses between 13000 and $21000 \mathrm{~cm}^{-1}$ can be generated. The coherent generation process minimizes the temporal jitter between the two pulses and allows to obtain a high time resolution of better than $0.3 \mathrm{ps}$ in excite and probe experiments.
\end{abstract}

\section{Introduction}

With the advent of picosecond light sources it became possible for the first time to investigate directly ultrashort relaxation processes in molecules. In numerous applications the pump and probe technique was used [1]. A first intense pulse excite the molecules, while a second, weaker and properly delayed pulse interrogates the transient response. Employing the excite and probe technique one derives two tunable pulses with a high degree of time resolution. Until now, several methods have been used to generate these pulses: (i) Synchronously pumped mode-locked dye lasers show a broad tunability in frequency with a pulse duration of $t_{\mathrm{p}} \sim 2$ to $3 \mathrm{ps}$ and a temporal jitter of 3 ps allowing a limited time resolution in most applications [2,3]. (ii) Mode-locked solid-state lasers (e.g. Nd : YAG or Nd : glass) have been used to produce widely tunable pulses by optical parametric amplification in nonlinear crystals. This process provides a smooth tunability [4,5] with a jitter $<0.5$ ps. (iii) Stimulated Raman scattering of pulses from solidstate lasers allows to shift frequencies by exchanging the Raman liquid. Using the processes (ii) and (iii) difficulties arise, when different frequencies are required for the excitation and probing beams. Using two frequency translators in a parallel configuration the time resolution is limited to approximately $1 \mathrm{ps}$.
In this letter we introduce a new experimental technique to generate picosecond pulses over a wide frequency range allowing a high time resolution of better than $0.3 \mathrm{ps}$ in excite and probe experiments. Pulses from a picosecond pump laser generate two frequency shifted pulses by stimulated and subsequent coherent Raman scattering. Due to the specific gener. ation process the two pulses are highly synchronized in time. A cross-correlation measurement and an excite and probe experiment demonstrate the good time resolution of the presented technique.

\section{Principles of operation}

The schematic of the experimental system is shown in fig. 1. A powerful second harmonic pulse from a mode-locked Nd : glass laser system $\left(t_{\mathrm{p}}=4 \mathrm{ps}, \widetilde{\nu}_{\mathrm{L}}=\right.$ $18990 \mathrm{~cm}^{-1}$ ) enters from the left. At the beam splitter BS1 the pulse is divided into two parts (amplitudes $E_{\mathrm{L} 1}$ and $E_{\mathrm{L} 2}$ ). The upper beam, $E_{\mathrm{L} 1}$, is focussed into the Raman cell, generator 1, generating a Stokes shifted pulse $\left(E_{\mathrm{S}}\right)$ by transient stimulated Raman scattering. Due to the transient and highly nonlinear stimulated Raman process the Stokes pulse has a shorter duration than the exciting laser pulse and has rapidly decaying wings [6]. The frequency $\nu_{\mathrm{S}}$ of the Stokes pulse $E_{\mathrm{S}}$ can be changed by using different generator 


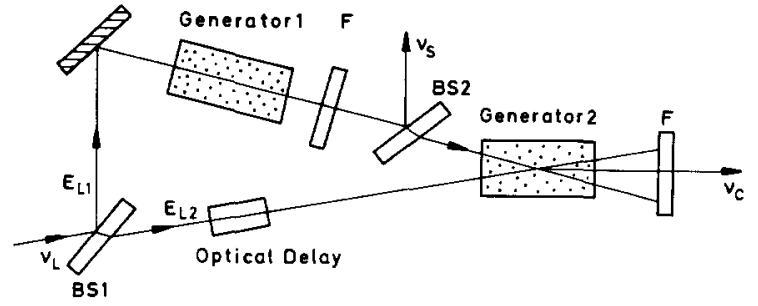

Fig. 1. Schematic of the set-up used to generate frequency shifted pulses by stimulated Raman scattering in generator 1 and subsequent coherent scattering in generator 2. Beam splitters BS1 and BS2, bandpass filters F. The two pulses at frequencies $\nu_{\mathrm{S}}$ and $\nu_{\mathrm{C}}$ are highly synchronized in time.

liquids $\left(\nu_{\mathrm{S}}=\nu_{\mathrm{L} 1}-\nu_{\mathrm{V} 1}\right.$, where $\nu_{\mathrm{V} 1}$ is the frequency of the vibrational mode with the highest Raman gain). The bandpass filter $F$ removes the laser light, while transmitting the stimulated pulse. Part of the Stokes pulse is extracted at the beam splitter BS2 and may be used in the subsequent excite and probe experiment, e.g. as an exciting pulse.

The second part of the incoming laser pulse which is transmitted through the beamsplitter BS1 passes an optical delay and is focussed into the Raman cell, generator 2. Here a second transient stimulated Raman process occurs: Simultaneously with a stimulated Stokes pulse $E_{\mathrm{S} 2}$ (not used further on) a coherent vibration $Q$ is produced. Coherent scattering of the Stokes pulse $E_{\mathrm{S}}$ from generator 1 at the coherent amplitude $Q$ (oscillating at frequency $\nu_{\mathrm{V} 2}$ ) creates the probe pulse $E_{\mathrm{C}}$. Using the appropriate phase-matching condition $\Delta k=0$ we may select either the Stokes or the anti-Stokes pulse at the frequencies $\nu_{\mathrm{CS}}=$ $\nu_{\mathrm{S}}-\nu_{\mathrm{V} 2}$ or $\nu_{\mathrm{CA}}=\nu_{\mathrm{S}}+\nu_{\mathrm{V} 2}$, respectively [7].

$\Delta k_{\mathrm{S}}=k_{\mathrm{S}}-k_{\mathrm{L} 2}+k_{\mathrm{S} 2}-k_{\mathrm{CS}}$,

$\Delta k_{\mathrm{A}}=k_{\mathrm{CA}}-k_{\mathrm{L} 2}+k_{\mathrm{S} 2}-k_{\mathrm{S}}$,

$\boldsymbol{k}$ are the wave vectors for the different light fields in the generator 2: The incident light fields $E_{\mathrm{S}}, E_{\mathrm{L} 2}$ produce fields $E_{\mathrm{S} 2}$ and $E_{\mathrm{CS}}$ or $E_{\mathrm{CA}}$. The propagation directions of the various incoming beams have to be adjusted in order to obtain phase-matching $\Delta k=0$. $\Delta k_{\mathrm{S}}=0$ allows Stokes, $\Delta \boldsymbol{k}_{\mathrm{A}}=0$ anti-Stokes generation [7]. The amplitude $E_{\mathrm{C}}$ of the coherently produced Stokes or anti-Stokes pulse is proportional to $Q$ $\times E_{\mathbf{S}}$.

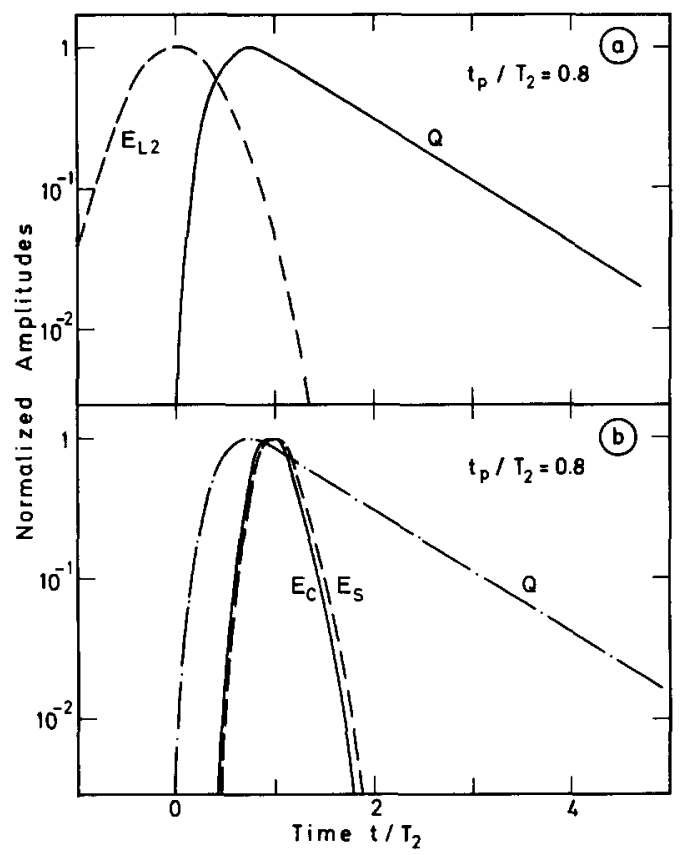

Fig. 2. (a) Calculated coherent amplitude $Q$ (solid curve) generated by the laser field $E_{\mathrm{L}_{2}}$ (broken curve) via transient stimulated Raman scattering. (b) Calculated coherent Raman scattering in generator 2. The incoming light field $E_{S}$ (broken curve) coherently interacts with the material excitation $Q$ (dash-dotted curve) which has been produced by the laser field $E_{\mathrm{L}_{2}}$. The coherently generated field $E_{\mathrm{C}}$ (solid curve) is synchronized in time with $E_{\mathrm{S}}$.

The evolution of the coherent scattering process is presented in fig. 2. Fig. 2a shows the generation of the coherent vibration $Q$ by the incident light pulse $E_{\mathrm{L} 2}$ via transient stimulated Raman scattering. The coherent amplitude $Q$ rises rapidly during the exciting pulse $E_{\mathrm{L} 2}$. At later times $Q$ decays exponentially with the dephasing time $T_{2}$. Fig. $2 \mathrm{~b}$ demonstrates the coherent scattering of the pulse $E_{\mathrm{S}}$ (broken curve) at the vibration $Q$ (dash-dotted curve). The coherently produced pulse $E_{\mathrm{C}}$ (solid curve in fig. 2b) has approximately the same shape as the pulse $E_{\mathrm{S}}$. It has the same rapidly decaying wings and is synchronized in time with the Stokes pulse $E_{\mathrm{S}}$. The energy of the coherently scattered light can be optimized by adjusting the optical delay (see fig. 1). In order to reject the laser pulse and the stimulated Stokes pulse transmitted through generator 2 a second bandpass filter $F$ is used. Under specific conditions e.g. $\nu_{\mathrm{C}} \approx \nu_{\mathrm{L}}$ or $\nu_{\mathrm{C}} \approx \nu_{\mathrm{S} 2}$, 
it may be favorable to use polarizers to suppress $E_{\mathrm{L} 2}$ and $E_{\mathrm{S}}$.

The properties of the two synchronized pulses $E_{\mathrm{S}}$ and $E_{\mathrm{C}}$ are as follows: Both pulses have a similar duration $t_{\mathrm{pS}}=t_{\mathrm{pC}} \approx 0.5 t_{\mathrm{p}}$. The energy of the pulse $E_{\mathrm{S}}$ is determined by the conversion efficiency in generator 1 . An energy conversion of $5 \%$ may be obtained without considerable loss of time resolution due to saturation processes [8]. Pulse $E_{C}$ is weaker than pulse $E_{\mathrm{S}}$ due to the coherent scattering process and may reach $5 \%$ of the energy of $E_{\mathrm{S}}$.

Of special interest is the broad spectral tunability of the two pulses: The frequency of the stronger pulse $E_{\mathrm{S}}$ is determined by generator 1 and the laser frequency $\nu_{\mathrm{L}}$ to be $\nu_{\mathrm{S}}=\nu_{\mathrm{L}}-\nu_{\mathrm{V} 1}$. It can be varied using transparent molecular liquids from $\nu_{\mathrm{S}} \sim \nu_{\mathrm{L}}$ to $\nu_{\mathrm{S}} / c=\nu_{\mathrm{L}} / c-3500 \mathrm{~cm}^{-1}$. A large variety of frequencies $\nu_{\mathrm{C}}$ may be obtained for the coherent pulse $E_{\mathrm{C}}$ by the independent choice of the two liquids in generators 1 and 2: $\nu_{\mathrm{C}}=\nu_{\mathrm{S}} \pm \nu_{\mathrm{V} 2}=\nu_{\mathrm{L}}-\nu_{\mathrm{V} 1} \pm \nu_{\mathrm{V} 2}$.

Fig. 3 shows - as an example - the many lines obtained from one laser and four Raman liquids (aceton, deuterated aceton, benzene, and carbontetrachloride). One has four Stokes frequencies, $\nu_{\mathrm{S}} / c=18531 \mathrm{~cm}^{-1}$, $17998 \mathrm{~cm}^{-1}, 16883 \mathrm{~cm}^{-1}$, and $16065 \mathrm{~cm}^{-1}$, and 27 possibilities for frequency $\nu_{\mathrm{C}}$ extending from $13140 \mathrm{~cm}^{-1}$ to $21456 \mathrm{~cm}^{-1}$. Using additional pump-

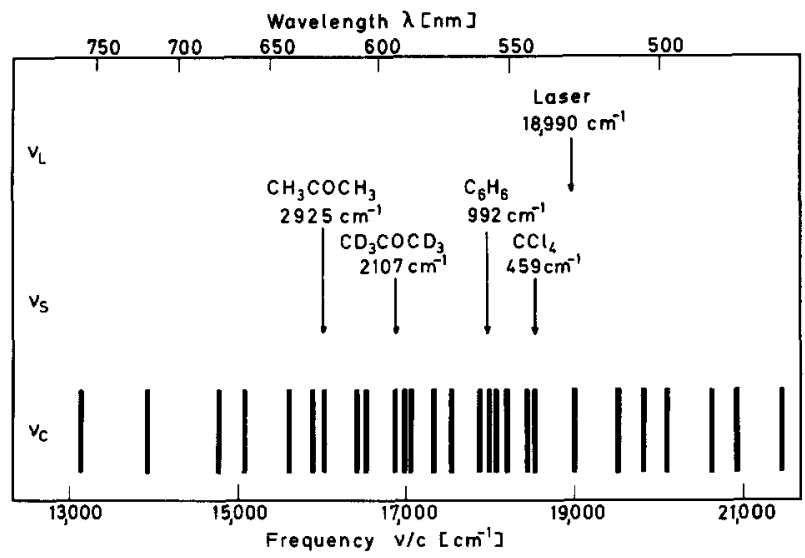

Fig. 3. Frequencies obtained when only four Raman active liquids are used in generator 1 and 2 . Four frequencies $\nu_{\mathrm{S}}$ may be obtained by stimulated Raman scattering in generator 1 , but 27 positions are found for the coherently scattered pulse at $\nu_{\mathrm{C}} . \nu_{\mathrm{L}} / c=18990 \mathrm{~cm}^{-1}$ is the frequency of the pumping laser pulse. ing frequencies (e.g. the fundamental or the third harmonic of the Nd : glass laser), the frequency range may be extended from the near UV to the near infrared region.

\section{Experimental}

Two examples demonstrate the favorable temporal properties of the frequency shifted pulses: (i) We show measurements of the cross-correlation function between the two pulses $E_{\mathrm{S}}$ and $E_{\mathrm{C}}$ and demonstrate the time resolution obtained for the measurement of rapidly decaying excitations. (ii) The build-up of a rapid absorbance change is studied and an upper limit for the rise time (the experimental resolution) is given.

In the first experiment we used carbontetrachloride in the first and acetone in the second generator cell. Consequently, we obtained the following frequencies: $\nu_{\mathrm{S}} / c=18531 \mathrm{~cm}^{-1}$ and $\nu_{\mathrm{CS}} / c=15606 \mathrm{~cm}^{-1}$. We have measured the cross-correlation function using background-free sum frequency signal generation in a KDP crystal. The experimental results are shown in fig. 4. Each experimental point is averaged over 15 individual measurements. The experimental

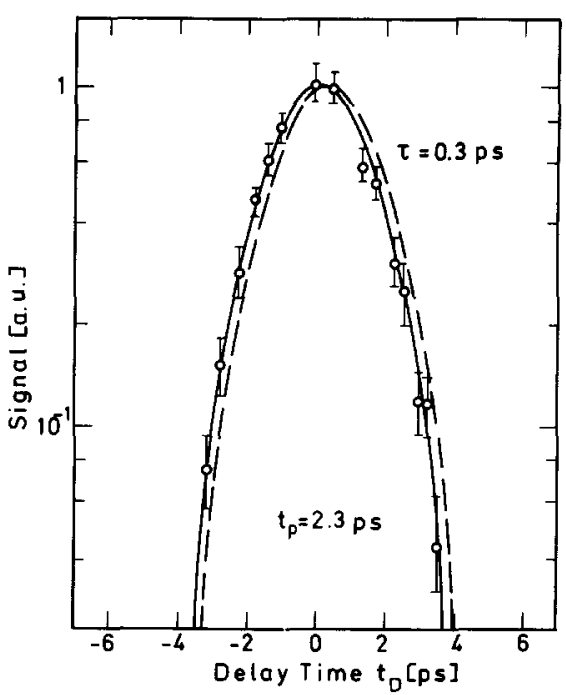

Fig. 4. Cross-correlation trace (circles and solid curve) between pulses at $\nu_{\mathrm{S}} / c=18531 \mathrm{~cm}^{-1}$ and $\nu_{\mathrm{C}} / c=15606 \mathrm{~cm}^{-1}$. Broken curve: Calculated absorbance change for a fictitious probing experiment, where a molecular excitation decays with $\tau=0.3$ ps. 
points are close to a gaussian shaped function (solid line). Assuming the same duration $t_{\mathrm{p}}$ of the two pulses $E_{\mathrm{S}}$ and $E_{\mathrm{C}}$ we calculate $t_{\mathrm{p}}=2.3$ ps. Note that we started with laser pulses of $4 \mathrm{ps}$, so that a considerable shortening is observed. The cross-correlation function shows steep nonexponential wings indicating that the jitter between the two pulses is negligible. In order to demonstrate the possibilities of the presented technique, we have calculated the signal curve one would expect for the following model system: The first pulse induces a molecular excitation showing a transient absorbance change. The rapidly decaying molecular excitation (decay time $\tau$ ) is probed by the second pulse: Using a decay time of $\tau=0.3$ ps we obtain the broken curve. This indicates a time resolution for this type of experiment of approximately $0.3 \mathrm{ps}$.

In the second experiment we use carbontetrachloride in both Raman generators leading to $\nu_{\mathrm{S}} / c=18531$ $\mathrm{cm}^{-1}$ and $\nu_{\mathrm{CS}} / c=18072 \mathrm{~cm}^{-1}$. Both frequencies are close to the absorption peak of Rhodamine B dissolved in methanol. The stronger pulse $E_{\mathrm{S}}$ is used to bleach the absorption of the dye while the second, much weaker pulse $E_{\mathrm{C}}$ monitors the absorbance decrease as a function of time in an improved excite and probe experiment [9]. Fig. 5 shows the experimental

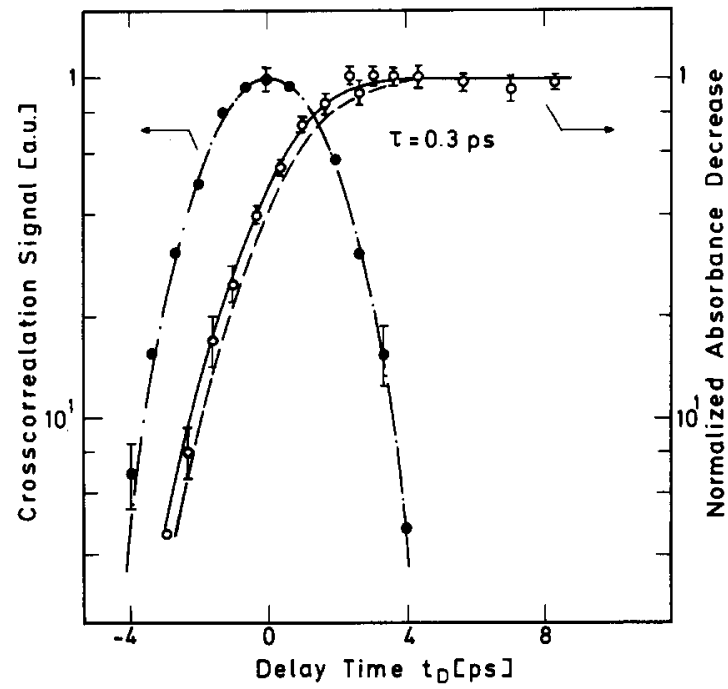

Fig. 5. Absorbance change of Rhodamine B (in methanol) as a function of delay time (open circles). Cross-correlation trace of exciting and probing pulses (solid points, dash-dotted curve). The solid and broken curves are calculated using a rise-time $\tau$ of the absorbance change of $\tau=0$ and $\tau=0.3 \mathrm{ps,}$ respectively.

results: The open circles indicate the absorbance decrease measured by the second pulse. During the pumping pulse $E_{\mathrm{S}}$ the signal rises and reaches a constant value at larger delay times. The experimental points accurately follow the integral (solid curve) of the cross-correlation curve (solid points, dash-dotted curve). From this experiment we obtain for the Rhodamine B molecules: (i) the absorption decreases without time delay as one would expect for the depopulation of the ground state, (ii) the population of the excited state remains constant during the observation time of $10 \mathrm{ps.} \mathrm{It} \mathrm{decays} \mathrm{with} \mathrm{the} \mathrm{well-known}$ lifetime of several nanoseconds. The presented example may be used to test a more general case: The first pulse $E_{\mathrm{S}}$ populates a molecular level which rapidly decays (decay time $\tau$ ) in a long-lived species. The absorption change of the final species is monitored by the pulse $E_{\mathrm{C}}$. For $\tau=0$ the observed absorbance change would follow the solid curve in fig. 5 . $\tau=0.3$ ps gives the broken curve which is well separated from the experimental points (open circles). This again demonstrates the high time resolution $(0.3 \mathrm{ps})$ for this type of experiment.

\section{Conclusions}

We have presented a new method to produce frequency shifted pulses which are well synchronized in time. The experimental set-up is easily aligned consisting of a few elements only. Using one pumping laser it is possible to obtain frequencies over a wide spectral range by exchanging two Raman liquids. The main properties of the pulses are summarized in table 1 . Short pulses $\left(t_{\mathrm{p}} \approx 2.3 \mathrm{ps}\right.$ ) with neglectable jitter allow to obtain a high time resolution of approximately $0.3 \mathrm{ps}$. Due to the conversion efficiencies of several

Table 1

Parameters of the pulses generated by stimulated and coherent Raman scattering

Pulse duration

Time resolution

Energy conversion in generator 1

Energy conversion in generator 2

Spectral bandwidth
$2.3 \pm 0.2 \mathrm{ps}$

$0.3 \mathrm{ps}$

between $2 \%$ and $5 \%$

$\approx 5 \%$.

$\lesssim 20 \mathrm{~cm}^{-1}$ 
percent the arrangement is well suited for pump and probe experiments, e.g. for recording the transient transmission changes of electronic transitions. The applications of the presented technique for the study of rapid transmission changes in biological systems are in progress and will be published elsewhere.

\section{Acknowledgement}

The authors acknowledge valuable contributions and stimulating discussions with Professor W. Kaiser.

\section{References}

[1] See for example: Picosecond phenomena III, Springer Series in Chemical Physics, Vol. 23, eds. K.B. Eisenthal, R.M. Hochstrasser, W. Kaiser and A. Laubereau (Springer Verlag, Heidelberg, 1982).
[2] J. Kuhl and D. von der Linde, in: Picosecond phenomena III, Springer Series in Chemical Physics, Vol. 23, eds. K.B. Eisenthal, R.M. Hochstrasser, W. Kaiser and A. Laubereau (Springer Verlag, Heidelberg, 1982) p. 201.

[3] J.M. Clemens, J. Najbar, I. Bronstein-Bonte and R.M. Hochstrasser, Optics Comm. 47 (1983) 271.

[4] A. Seilmeier and W. Kaiser, Appl. Phys. 23 (1980) 113.

[5] F. Wondrazek, A. Seilmeier and W. Kaiser, Appl. Phys. B32 (1983) 39.

[6] R.L. Carman, F. Shimizu, C.S. Wang and N. Bloembergen, Phys. Rev. A2 (1970) 60.

[7] A. Laubereau and W. Kaiser, Rev. Mod. Phys. 50 (1978) 607 ;

W. Zinth, H.J. Polland, A. Laubereau and W. Kaiser, Appl. Phys. B26 (1981) 77.

[8] W.H. Lowdermilk and G.I. Kachen, Optics Comm. 18 (1976) 68.

[9] H.J. Polland, T. Elsaesser, A. Seilmeier, W. Kaiser, M. Kussler, N.J. Marx, B. Sens and K.H. Drexhage, Appl. Phys. B32 (1983) 53. 